\title{
Imaging the Structural Evolution in Nanocrystalline Metals during Mechanical Deformation
}

\author{
C. Kübel ${ }^{1,2}$, A. Kobler ${ }^{1,3,4}$, A. Kashiwar ${ }^{1,3}$, H. Hahn ${ }^{1,3}$ \\ ${ }^{1 .}$ Karlsruhe Institute of Technology (KIT), Karlsruhe, Germany \\ 2. Karlsruhe Nano Micro Facility (KNMF), Karlsruhe, Germany \\ 3. Technical University Darmstadt (TUD), Darmstadt, Germany \\ 4. ZEISS Microscopy, Peabody, MA, USA
}

Most of our current understanding of the deformation mechanisms active in nanocrystalline (nc) metals stems from in situ deformation experiments on bulk materials using x-ray diffraction (XRD). However, XRD cannot directly resolve the local deformation processes. For a local analysis, these processes are traditionally investigated using BF/DF-TEM. However, varying contrast due to local orientation changes, bending and defects during in situ BF-TEM straining experiments make an accurate interpretation for nanometer sized grains difficult. On the other hand, automated crystal orientation mapping (ACOM-TEM) [1] allows to identify the crystallographic orientation of all crystallites with sizes down to around $10 \mathrm{~nm}$, well below the limit of electron back scatter diffraction (EBSD). Performing ACOM-TEM in $\mu$ p-STEM mode allows to image grain orientations in situ during straining inside a TEM $[2,3]$. This combination was key to a new data evaluation based on orientation maps. By tracking individual crystallites through a straining series the change of their orientation can be evaluated in order to distinguish between local crystallite rotation and sample tilting/bending [2, 3]. In addition, twinning/detwinning and grain growth can be directly followed (Fig. 1, 2) and the automatic data evaluation leads to user independent quantitative statistical information such as grain size distribution and grain rotation statistics [3].

One of the challenges using ACOM-STEM for in situ experiments are crystallites with different orientations overlapping in projection. The overlapping crystallites lead to superimposed diffraction patterns that result in deviations during the matching procedure. Tilting a nc Pd thin film and tracking the changes using ACOM-STEM, these effects were analyzed and discussed in the context of sample bending during in situ experiments [4]. The challenges with sample bending do not only appear with ACOM-STEM, but are inherent problems for any TEM projection technique. However, using ACOM-STEM sample bending/tilting during an in situ series can be quantified and the effects considered for the interpretation of the results. In addition, the ' $180^{\circ}$ ambiguity problem' [5] does not only present a challenge within single orientation maps, but also has to be considered within in situ series, when comparing the orientation of a grain throughout a series. Therefore, we have developed an ambiguity filter for series of orientation maps analogous to the ambiguity filter in single orientation maps [3].

While ACOM-STEM is a powerful approach for in situ experiments on nanocrystalline materials, a major disadvantage is that the acquisition of one statistically meaningful orientation map takes around 30-60 min, putting serious limits to the stability of the in situ deformation setup. In particular, a detailed analysis of the mechanical data is difficult. In current experiments, we therefore combine BF/DF-STEM with ACOM analysis at a few selected states to increase the speed of the in situ straining experiments. This approach seems to be promising in order to study the Bauschinger effect in nanocrystalline metals, obtaining both reliable structural and mechanical data.

In this presentation, we will discuss the challenges of reliable structural and mechanical characterization and illustrate it using in situ straining of $\mathrm{nc} \mathrm{Pd}_{\mathrm{x}} \mathrm{Au}_{1-\mathrm{x}}$ thin films as example, looking 
at twinning, $\Sigma 9$ boundary formation, grain rotation and dislocation activity with respect to the Bauschinger effect [6].

\section{References:}

[1] E.F. Rauch, et al, Zeitschrift für Krist, 225 (2010), p. 103-109.

[2] A. Kobler et al, Ultramicroscopy 128 (2013), p. 68.

[3] A. Kobler et al, Beilstein J. Nanotechn 7 (2016), p.572.

[4] A. Kobler, C. Kübel, Ultramicroscopy 173 (2017), p. 84.

[5] E.F. Rauch, M. Véron, Microsc Microanal 19 (2013), p. 324.

[6] The authors acknowledge support by the Karlsruhe Nano Micro Facility (KNMF) for access to the electron microscopic equipment.
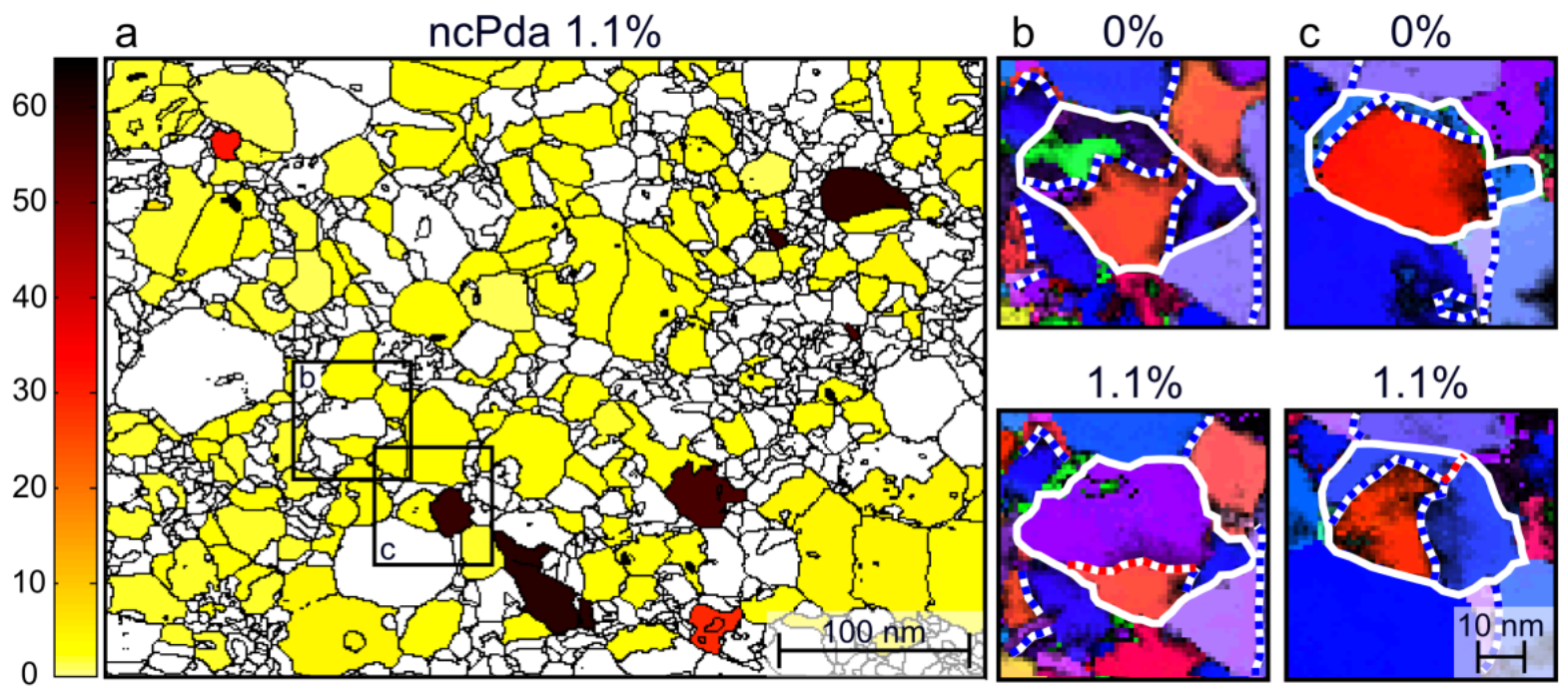

Figure 1. In situ tensile test of nc Pd combined with ACOM-STEM analysis: Crystallite rotations in the range of $0^{\circ}-65^{\circ}$ : a) Crystallite boundary map overlaid with the rotation of selected tracked crystallites for the $1.1 \%$ strain state. b,c) ACOM-STEM orientation maps of selected areas marked in (a). The $\Sigma 3$ boundaries are shown with white/blue dashed lines and $\Sigma 9$ boundaries with white/red dashed lines; the white boundary outlines the grain of interest.
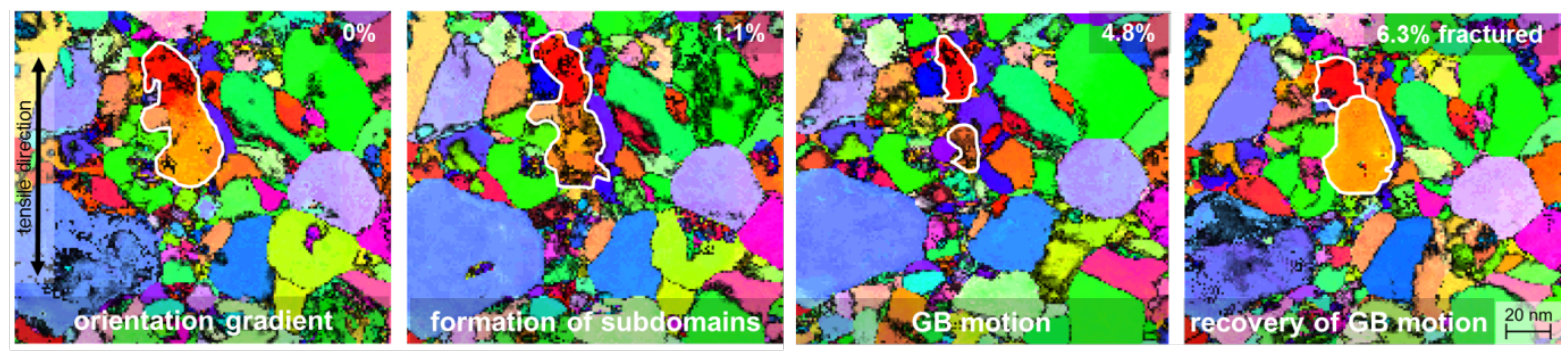

Figure 2. Partial recovery of plastic deformation during in situ tensile testing of $\mathrm{nc} \mathrm{Pd}$. For the highlighted grain, the ACOM maps show the development of sub-grain boundaries leading to the formation of separate grains with increasing strain, followed by partial recovery of the plastic deformation after fracture of the sample. 